\title{
Normes textuelles et construction d'une identité professionnelle
}

\author{
Le cas des chroniqueurs de la presse écrite \\ québécoise
}

Franz Meier, chargé de recherche et d'enseignement

Universität Augsburg

franz.meier@phil.uni-augsburg.de 
60 Dynamiques de professionnalisation en communication

\title{
Résumé
}

Les chroniqueurs de la presse écrite québécoise jouissent à l'heure actuelle d'une forte popularité, surtout en raison de l'originalité de leur écriture. Compte tenu de leur notoriété publique, nous cherchons à savoir s'ils ont développé une identité professionnelle qui diffère de celle des autres scripteurs en milieu journalistique. Dans cette optique, nous nous intéressons à la perception de certaines traditions textuelles, aussi appelées normes textuelles, aspect que nous traitons à partir d'un corpus d'entretiens semi-dirigés réalisés auprès de douze chroniqueurs qui écrivent pour des quotidiens à tirage élevé. Nous nous proposons d'analyser dans quelle mesure les chroniqueurs considèrent ces normes textuelles comme des constituants de leur identité professionnelle qui devraient régir leurs pratiques. Les résultats montrent que la place qu'accordent les chroniqueurs aux variations individuelles dans ces techniques d'écriture leur permet de se démarquer en tant qu'auteurs.

Mots-clés : normes textuelles, chroniqueurs, presse écrite québécoise, identité professionnelle

\begin{abstract}
Today, Quebec newspaper columnists are very popular, especially because of the originality of their writing. Given their popularity, the aim of this paper is to analyse if these columnists have developed a professional identity that differs from other writers in the journalistic community. To this end, we focus on the columnists' perception of certain text traditions, i. e. textual norms. Our analysis bases on a data collection of semi-structured interviews with twelve columnists who write for daily newspapers. We examine the extent to which these columnists consider different textual norms governing their daily writing as components of their professional identity. The results show that the scope these columnists give to individual variation in well-established text traditions allows them to stand out as authors.

Keywords: textual norms, columnists, Quebec daily newspapers, professional identity
\end{abstract}




\section{Le rôle des chroniqueurs dans la presse écrite québécoise}

Titulaires d'une rubrique publiée périodiquement dans un journal, les chroniqueurs sont des journalistes qui donnent leur opinion sur un sujet d'actualité lié à une section particulière - politique, finance, sport, etc. - ou sur un thème de leur propre choix (Agnès, 2008 ; Grevisse, 2008). À l'inverse des éditorialistes qui, en tant que représentants de la direction, défendent la position officielle du journal, les chroniqueurs sont généralement des journalistes employés qui peuvent aussi, en plus de la rédaction de leur chronique, écrire d'autres types d'articles, comme des nouvelles ou des reportages (Ross, 2005, p. 23). Par ailleurs, nombre de chroniqueurs tiennent également des blogues qui occupent une place importante sur le site web des journaux.

Même si le genre de la chronique n'existe que depuis une quarantaine d'années dans les quotidiens québécois, où il est d'abord apparu dans les sections du sport et des arts et spectacles (Picard, 1999, p. 36-37), il ne fait aucun doute que ses auteurs, incarnés par exemple par Pierre Foglia et Lysiane Gagnon à La Presse ou par Jean Dion et Josée Blanchette au Devoir, jouissent à l'heure actuelle d'une forte popularité. Parmi les raisons expliquant que «les lecteurs [...] se précipitent littéralement sur les chroniques » (Maltais, 2010, p. 34), on peut noter l'humeur et l'originalité de ces textes qui sont considérés, pour en croire plusieurs manuels de journalisme (Maltais, 2010 ; Noël, 2009), comme l'exemple d'une écriture particulièrement vivante et imagée. Conscients de cette notoriété publique, les éditeurs attribuent depuis quelque temps un rôle important aux chroniqueurs dans le marketing des quotidiens (Picard, 1999, p. 37), notamment pour diversifier le contenu des publications et ainsi fidéliser les lecteurs.

La popularité des chroniqueurs est un phénomène intéressant dans l'analyse de la dynamique de professionnalisation du secteur journalistique, c'est-à-dire lorsque l'on cherche à dégager les ruptures et continuités de la "culture d'une activité » (Ruellan, 1993, p. 31) propre aux journalistes. En effet, les chroniqueurs constituent un groupe de journalistes de plus en plus nombreux dans les salles de rédaction, évolution qui résulte essentiellement de l'apparition du journalisme dit de communication à partir des années 1970 et 1980 (Brin, Charron \& Bonville, 2004, p. 4). Dans une situation de concurrence accrue sur le marché des médias, les pratiques journalistiques sont désormais davantage animées par le souci d'établir et de maintenir des rapports de proximité et d'intersubjectivité avec les lecteurs, ce qui fait une plus grande place au commentaire. Dans ce contexte global, on observe la mise en scène des chroniqueurs comme scripteurs vedettes, ce qui semble induire une opposition avec les autres journalistes de la presse écrite dont la tâche consiste 
avant tout à rapporter fidèlement des évènements et les propos des personnes rencontrées.

C'est ainsi que nous cherchons à savoir si, compte tenu de leur popularité médiatique, les chroniqueurs ont développé une identité professionnelle spécifique, ou pour reprendre les mots d'Amossy (2010), un éthos collectif, qui leur permet de se différencier des autres scripteurs travaillant dans le champ journalistique (Bourdieu, 1996). À cet effet, nous nous proposons d'examiner la manière dont les chroniqueurs perçoivent certaines normes textuelles en usage en écriture journalistique et dans quelle mesure ils les considèrent comme des points de repère qui devraient orienter leurs pratiques dans la rédaction de chroniques. En ce sens, nous envisageons la conception qu'ont les chroniqueurs de ces normes textuelles comme un des paramètres qui contribue à la construction de leur identité professionnelle (Dubar, 1991). Cette contribution s'inscrit dans le cadre d'un travail antérieur (Meier, 2016) consacré à l'étude de la conscience linguistique des professionnels des médias écrits québécois, et ce, par l'analyse de normes textuelles, communicationnelles et linguistiques qui sous-tendent les productions journalistiques. Le corpus analysé était constitué de 39 entrevues semi-dirigées réalisées entre 2011 et 2013 auprès de 12 chroniqueurs, 16 enseignants de rédaction journalistique et 11 langagiers, c'est-à-dire des traducteurs et (ou) des réviseurs qui travaillent pour la plupart dans les services linguistiques des journaux.

\section{Les normes textuelles en tant que piliers de la professionnalisation journalistique}

Aux fins de notre analyse, nous définissons la notion fortement polysémique de norme comme une obligation voulue ou ressentie qui détermine le comportement langagier des individus. Cette définition s'inspire des propos du germaniste Gloy (1993) qui conçoit les normes comme des abstractions mentales de caractère plutôt implicite. Les normes explicites, quant à elles, sont des règles codifiées qui peuvent devenir des normes proprement dites si l'individu les intériorise comme une entité déontique (Gloy, 2004). Pour examiner plus particulièrement les normes textuelles régissant les pratiques journalistiques, nous nous proposons de revenir sur le modèle des traditions discursives proposé par Koch (1987). Ce modèle part du principe que toute énonciation est déterminée non seulement par des normes linguistiques propres à une langue particulière, mais aussi par des normes discursives ou textuelles relevant de traditions textuelles historiquement stabilisées qui font l'objet de changements permanents.

Ces traditions sont généralement définies comme des «techniques d'élaboration textuelle » (Aschenberg, 2003, p. 5) qui servent de " schémas ou modèles textuels » (Oesterreicher, 1997, p. 20) permettant aux locuteurs l'attribution d'un exemplaire textuel à une série historique de textes (Wilhelm, 2001, p. 470). D'un point de vue 
strictement normatif, ces traditions représentent des habitudes d'écriture socialement partagées et individuellement acquises qui créent un horizon d'attente réciproque par rapport aux pratiques des scripteurs (Oesterreicher, 1997). Parmi ces traditions de rédaction, Wilhelm (2001, p. 468) distingue différents degrés d'abstraction, à savoir les formules fixes et prédéfinies, les genres en tant que schémas textuels plus complexes et les univers de discours comme la littérature, l'expérience quotidienne, la science et la religion. Les traditions textuelles peuvent donc constituer des schémas polymorphes qui intègrent des composants très hétérogènes (Oesterreicher, 1997, p. 31).

Selon Ringoot et Utard (2005 \& 2009), ces traditions textuelles, et ici notamment les genres, jouent un rôle important dans la professionnalisation du milieu journalistique, c'est-à-dire dans la mise en place d'un mode de production propre aux journalistes. D'abord, elles contribuent, à partir des dernières décennies du XIX siècle, à une délimitation du journalisme avec d'autres groupes scripteurs relevant de différents univers discursifs, par exemple la littérature, démarcation qui semble particulièrement prononcée en journalisme nord-américain (Chalaby, 1998 ; Neveu, 2013). En outre, les traditions textuelles contribuent à une spécialisation et à une structuration interne de la pratique journalistique, ce qui va généralement de pair avec la codification des techniques professionnelles dans des manuels de journalisme et leur transmission dans des écoles ou à l'université. Cette formalisation de l'activité journalistique concerne non seulement la collecte et le traitement de l'information, mais aussi l'élaboration textuelle même. En d'autres termes, on aboutit à un journalisme de plus en plus standardisé où le talent individuel « cède la place à la méthode » (Ringoot \& Utard, 2005, p. 27), comme à celle du lead, le premier paragraphe où il faut répondre aux « cinq $\mathrm{W}$ » et au « $\mathrm{H}$ » (what/quoi, who/qui, when/quand, where/où, why/pourquoi et how/comment) ou encore à celle de la pyramide inversée, qui impose une structure textuelle qui va du plus au moins important (Sormany, 2011, p. 89).

Dans cette optique, ce sont les chroniqueurs qui semblent échapper le plus à cette dynamique, d'autant plus que l'écriture de chronique n'est pas enseignée dans les écoles de journalisme et qu'elle n'est que vaguement décrite dans les manuels. Dans les faits, la chronique y est reconnue comme « le genre journalistique le plus libre, le plus diversifié » (Sormany, 2011, p. 140), « le genre variable par excellence » (Ross, 2005, p. 22) qui demande essentiellement l'affirmation de la personnalité du journaliste qui l'écrit, et ceci même par le recours à des techniques d'écriture littéraires, comme c'était aussi le cas dans le nouveau journalisme des années 1960. Dans ce qui suit, nous nous pencherons d'abord sur la perception qu'ont les chroniqueurs de traditions textuelles standardisées et codifiées dans des ouvrages de référence. Par la suite, nous aborderons le point de vue qu'ils adoptent sur leurs propres pratiques rédactionnelles. En terminant, nous nous intéresserons à la 
64 Dynamiques de professionnalisation en communication

manière dont ils perçoivent les processus d'hybridation observables en écriture journalistique.

\section{Analyse}

De l'ensemble du corpus présenté plus haut, nous nous concentrons ici sur les propos tenus par les 12 chroniqueurs ( 7 hommes et 5 femmes), qui écrivent pour des quotidiens québécois à tirage élevé, à savoir La Presse, Le Devoir et Le Soleil, où ils couvrent les sections consacrées aux actualités politiques, aux questions éthiques et sociales ainsi qu'aux arts et spectacles. Notre échantillon ne tient compte ni de chroniqueurs qui travaillent pour des tabloïds, ni de chroniqueurs-pigistes qui publient à l'occasion des textes en dehors de leur profession régulière, bien que ceux-ci soient aussi nombreux dans le paysage de la chronique québécoise. Tous originaires du Québec, les chroniqueurs interrogés ont un âge moyen de 50,4 ans, ce qui diffère des résultats obtenus lors d'enquêtes antérieures (Pritchard \& Sauvageau, 2008, p. 95), qui montrent que les journalistes en exercice au Québec, tous médias confondus, sont plus jeunes de dix ans (41,8 ans). Cet écart pourrait s'expliquer par le fait que les chroniqueurs comptent parmi les journalistes qui ont le plus d'expérience professionnelle. En effet, selon plusieurs témoins de notre étude, nombreux sont ceux qui ont d'abord travaillé comme journalistes d'information et qui, se démarquant par l'originalité de leur pensée et de leur écriture, sont ensuite devenus chroniqueurs. Tous les témoins indiquent avoir une ou plusieurs formations universitaires à leur actif, surtout en sciences sociales (histoire, sociologie, ethnologie, droit et sciences politiques) et, dans une moindre mesure, en lettres, mais seuls cinq ont étudié le journalisme, et ce tant dans des universités québécoises (Université Laval, Université de Montréal, Université du Québec à Montréal) que dans des écoles de journalisme françaises (Ecole supérieure de journalisme de Lille). À l'inverse, Pritchard et Sauvageau (2008, p. 95), qui ne distinguent pas entre chroniqueurs et autre journalistes, constatent que seuls $62,8 \%$ des professionnels des médias détiennent un diplôme universitaire et que $59,4 \%$ des journalistes ont étudié dans ce domaine.

L'entrevue comprend une vingtaine de questions, dont plusieurs assertions provenant du milieu des médias québécois que nous avons proposés aux témoins pour ensuite leur demander leur opinion. Deux assertions et une question qui touchent des aspects liés aux normes textuelles ont été retenues dans la présente analyse, à savoir «Jean de Bonville, de l'Université Laval, observe une tendance vers une écriture journalistique plus individualisée où les journalistes cherchent systématiquement à se distinguer les uns des autres sur le plan stylistique ", «Le journaliste Daniel Raunet critique le fait que la presse québécoise mélange ouvertement les genres" et "Les chroniqueurs peuvent-ils servir de modèle en écriture journalistique?». 


\subsection{La perception des normes textuelles standardisées}

Dans le cadre de ces questions et assertions, les chroniqueurs se sont prononcés sur des normes textuelles standardisées qui devraient organiser le contenu des articles, par exemple le lead ou la pyramide inversée. L'examen des réponses révèle que les témoins perçoivent ces techniques comme des repères déontiques qu'ils associent plutôt à l'écriture de textes d'information, où ils devraient s'appliquer presque inconsciemment. Les témoins considèrent donc ces techniques comme des automatismes qui relèvent d'un système normatif parfaitement intériorisé par les journalistes écrivant des textes à visée informative, ce qui n'est pas le cas, du moins pas dans une proportion aussi importante, en rédaction de chronique. Les répondants établissent ainsi une catégorisation professionnelle, que l'on trouve aussi dans nombre de manuels de journalisme (Maltais, 2010, p. 37), basée sur la distinction entre des genres factuels et d'opinion. ${ }^{19}$

Les chroniqueurs invoquent souvent le contrat de communication (Charaudeau, 2002, p. 141) qu'ont établi journalistes et lecteurs à l'égard de traits caractéristiques des textes d'information pour justifier l'importance de ces normes standardisées. De ce point de vue communicationnel, ces textes exigeraient une structuration préétablie pour que « tous les éléments, la démonstration, [...] ce qui arrive, ce qui n'arrive pas, $[\ldots]$ pourquoi, qui, quand, à quelle heure et pour qui » $(\mathrm{P} 35)^{20}$ soient facilement compréhensibles :

FM : Et l'écriture de nouvelles, vous la trouvez trop standardisée (?)

P35 : Mais je pense qu'il faut qu'elle le soit, honnêtement, parce que là, on est vraiment dans un message clair, pur, il faut que les gens comprennent tout, on a intérêt que ce soit standardisé (P35)

Un autre argument récurrent pour prouver la nécessité de ces normes standardisées est celui de l'espace accordé aux journalistes pour produire un message cohérent. Ainsi, les chroniqueurs évoquent le nombre croissant de contenus à transmettre, ce qui obligerait les journalistes de l'information à recourir à une organisation formatée pour fournir les éléments essentiels en quelques lignes :

\footnotetext{
${ }^{19}$ Signalons que cette classification n'est pas définie de manière homogène dans les manuels et qu'elle inclut parfois une troisième classe de genres, ceux d'explication (Ross, 2005, p. 6).

${ }^{20}$ Dans les exemples cités, les tours de parole de l'enquêteur ont été marqués par $F M$ et ceux des participants, par un numéro de code suivant le modèle PXX, où la variable XX indique le numéro de l'entretien. La transcription évite dans la mesure du possible la ponctuation. Les virgules sont néanmoins utilisées pour indiquer la fin d'un segment de la chaine parlée, une pause est indiquée par ... et une séquence omise par [...].
} 
66 Dynamiques de professionnalisation en communication

Il y a de plus en plus de nouvelles et il y a pas nécessairement de plus en plus de temps d'antenne pour les exposer, donc, la bataille pour l'espace, elle est vitale dans le journalisme écrit, [...] c'est pas le temps des humeurs personnelles (P09)

La question du format court se pose différemment en chronique, où les scripteurs disposent certes aussi d'un espace limité, mais, contrairement aux journalistes d'information, il s'agit d'un « espace garanti [...] qui leur est réservé » (Picard, 1999, p. 46). Dans cette perspective, il est compréhensible que les chroniqueurs perçoivent les techniques du lead et de la pyramide inversée comme des normes à suivre surtout en journalisme d'information, même s'ils ne considèrent pas toujours qu'un alignement parfait sur ces schémas est une pratique souhaitable. Pour plusieurs chroniqueurs, ce n'est que la dépêche d'agence qui devrait strictement respecter ces normes standardisées, alors que les textes de nouvelle figurant dans les quotidiens pourraient, voire devraient, s'écarter du modèle dit agencier. En d'autres termes, le degré d'acceptabilité d'une écriture d'information moins standardisée dépend significativement du genre à visée informative. Il semble donc que les chroniqueurs établissent une hiérarchisation professionnelle à l'intérieur du groupe des journalistes d'information, attribuant une plus grande autonomie rédactionnelle aux scripteurs de nouvelle qu'aux agenciers :

Il faut aussi dans un journal se distinguer des textes d'agences de presse, pour moi, un texte d'agence de presse, c'est [...] la quintessence de ... de ... on va directement au but, on est dans [...] la simplicité, alors que ... je trouve qu'un journaliste, quand même, dans un journal pour se distinguer de cette espèce de norme-là, il faut aller un peu plus loin, peut-être dans l'écriture, soigner un peu plus tout ça (P38)

Compte tenu de la distinction établie entre les différents genres factuels, il est peu surprenant que plusieurs chroniqueurs comparent l'écriture agencière à un « moule » (P19 \& P37) duquel semble sortir une écriture jugée « un peu pauvre» (P25) ou " homogène et hermétique » (P34). Le dernier témoin affirme même que « c'est un calvaire, faire une dépêche d'agence, c'est [...] trop facile et c'est [...] ennuyant, parce que [...] c'est vraiment juste une succession de sujets, verbes, compléments » (P34). Le répondant finit par reprocher aux écoles de journalisme de trop se concentrer sur la circulation des techniques du « modèle classique agencier » (P34), qui est considéré comme le « modèle de formation quasi unique » (P34) :

Les journalistes [...] ont tous été formés à peu près au même endroit, ils viennent du même moule et [...] ça paraît, les jeunes journalistes qui rentrent ici, ça paraît, tu sais, quand tu lis ce qu'ils font [...][le journalisme] est une profession qui étrangement cultive le conformisme ... donc [...] celui qui essaie de se distinguer là-dedans devient forcément le mouton noir, et il y a pas de journaliste qui aime jouer le rôle du mouton noir, [...] dans la nouvelle brute, les journalistes essaient 
tous $[. .$.$] de faire la même chose ... ils essaient pas trop de se distinguer ... ils vont$ davantage chercher à se distinguer par la nouvelle exclusive qu'ils vont être capables d'aller chercher, mais pas par le style (P34)

En somme, les chroniqueurs se distancient en quelque sorte de la dynamique de professionnalisation journalistique dans la mesure où ils estiment que la standardisation des techniques rédactionnelles risque d'effacer toute variation individuelle en écriture d'information. En critiquant ces techniques, les chroniqueurs se présentent comme appartenant à un groupe distinct de celui des autres membres du champ journalistique, surtout des journalistes de l'information.

\subsection{La perception des normes textuelles en écriture de chronique}

Il n'est pas étonnant que les répondants perçoivent la chronique comme le genre qui laisse le plus de liberté rédactionnelle aux journalistes. Ils insistent sur la nécessité de mettre en évidence leur personnalité par un style individuel (Gauger, 1995, p. 212), c'est-à-dire par une manière d'écrire typique à chaque chroniqueur :

On parle plus [...] individualiste dans les chroniques, c'est ça une chronique, ils nous le demandent à nous, puis c'est mon nom et [...] évidemment, $[\ldots]$ je vais essayer d'avoir un style, donc, je trouve que c'est une sorte de vérité de La Palice et si on peut se distinguer effectivement au plan stylistique, c'est pas qu'on essaie de le faire, c'est qu'on est différent, on vient chacun de milieux différents, on a une éducation, donc [...] on espère que ... parce que c'est comme ... tu lis un texte et tu dis, eh bien oui, évidemment, ça, c'est Foglia, puis j'espère que tu dis ah bien ça, c'est [...], oui, c'est important (P26)

S'il est vrai que les chroniqueurs s'accordent une très grande marge de manœuvre sur le plan stylistique, plusieurs répondants, surtout les chroniqueurs du secteur de l'actualité générale, précisent que leurs textes sont structurés non pas de façon arbitraire, mais plutôt en fonction de certains principes de rédaction. Ces témoins soulignent surtout l'importance des parties factuelles de leurs chroniques, car ces passages contribuent à justifier leurs opinions. Dans cette perspective, le répondant cité ci-dessous décrit sa chronique comme un enchaînement de passages factuels et de prises de position personnelles qui, de leur côté, doivent s'appuyer sur les informations données auparavant :

Je fais pas une chronique purement d'humeur ou [...] d'émotions, [...] je veux faire une chronique qui soit informative et où je peux soutenir un raisonnement d'opinion [...] à partir de faits établis, d'intérêt public et ensuite, on peut être d'accord ou pas avec ma conclusion, mais j'appuie sur des faits ... donc c'est ça, c'est qu'on [...] puisse [...] suivre à la trace de mon raisonnement (P27) 
Dans un même ordre d'idées, un autre témoin décrit la rédaction des différentes séquences de ses chroniques au moyen de la dichotomie entre automatismes et efforts. $^{21}$ Or, rédiger des passages d'information est perçu comme une pratique plutôt mécanique qui se fonderait sur des normes standardisées et complètement intériorisées, alors que l'écriture de passages d'opinion demanderait, faute de schémas textuels préétablis, plus d'efforts :

Quand on fait les aspects factuels, c'est plus facile, avec l'expérience, on peut y arriver assez rapidement, [...] il y a des expressions [...] qui sont usuelles pour résumer des situations, et c'est sûr que les portions où on fait de l'opinion dans [...] une chronique, [...] moi, il y a toujours un aspect très factuel dans mes chroniques, donc, mais la portion où là, c'est opinion et émotion quand nécessaire, c'est ça qui prend le plus de temps à écrire (P36)

Cette citation va dans le même sens de ce qu'Oesterreicher (1997) affirme à propos des traits caractéristiques des traditions textuelles. Elle illustre que les répondants perçoivent la chronique comme un schéma complexe et polymorphe qui peut réunir des éléments très hétérogènes. Du point de vue de la réflexion sur l'identité professionnelle des chroniqueurs, il est intéressant de voir que les témoins affirment aussi recourir à des structures textuelles qu'ils situent d'abord en journalisme d'information. Les répondants considèrent donc la rédaction de chroniques comme une activité peu réglementée qui permet des emprunts à d'autres traditions textuelles. Ainsi, un chroniqueur affirme que «vous pouvez faire même une $[\ldots]$ chronique $[\ldots]$ sous forme de [...] poème, un sonnet, sous forme de texte $\mathrm{d}$ 'humour, une lettre imaginaire, $[. .$.$] il y a pas [\ldots]$ de règle d'exclusion » $(\mathrm{P} 09)$.

En outre, plusieurs témoins caractérisent leur propre pratique rédactionnelle en l'opposant à d'autres genres provenant du domaine du commentaire. D'une part, un chroniqueur déclare reconnaitre peu de différences entre la chronique et le blogue, la longueur textuelle mise à part :

Des fois, je me dis c'est quoi la différence [...] entre un blogue puis une chronique (?) je pense qu'il y en a plus, sinon qu'un blogue, on va mettre ... ça va être deux paragraphes, une chronique, c'est plus long, on va développer davantage (P26)

D'autre part, les répondants tiennent à se distinguer des éditorialistes en soulignant que la chronique n'engage que son signataire en tant qu'émetteur d'opinions personnelles, alors que les éditorialistes représentent le journal dans son ensemble.

\footnotetext{
${ }^{21}$ Seiler $(2009$, p. 33) considère les remarques faites par les locuteurs au sujet des automatismes et des efforts comme des indicateurs potentiels de l'existence de normes.
} 
Les témoins avancent donc un critère de différenciation qui concerne moins des aspects textuels que le statut professionnel des personnes qui écrivent ces textes :

\begin{abstract}
L'éditorial, c'est vraiment une tradition anglo-saxonne, c'est la page qui appartient au propriétaire et qui reflète l'opinion du propriétaire, c'était ça au début, alors là, $[\ldots]$ on est plus ou moins libre de notre opinion, il faut faire un consensus le matin autour de la table éditoriale, parce que chroniqueur, on écrit ce qu'on veut, ou presque, dans les limites de l'acceptable et de ce que les patrons acceptent, on ne peut pas appeler à la révolution et à mettre le feu à Montréal là, mais ... on reflète notre propre opinion et non celle du journal (P19)
\end{abstract}

On constate que les normes textuelles occupent une place importante dans la construction de l'identité professionnelle des chroniqueurs, même si ces normes constituent en grande partie des principes de rédaction très généraux qui ne sont pas propres à la chronique. En effet, les chroniqueurs se montrent très ouverts à l'utilisation, voire à l'amalgame, de différentes normes textuelles, attitude qui ne les empêche toutefois pas de vouloir se distinguer des autres journalistes du commentaire, tout particulièrement des éditorialistes.

\title{
3.3. La perception des processus d'hybridation générique en écriture journalistique
}

Si les répondants semblent avoir tendance à combiner diverses traditions rédactionnelles dans leurs propres chroniques, ils ne sont toutefois pas «très favorable[s]» (P38) à la transgression des genres journalistiques qu'on peut généralement observer dans les autres productions en presse écrite (Burger, 2005, p. 206). Ainsi, la grande majorité affirment y reconnaitre « un mélange des genres plus grand qu'on devrait l'avoir » (P27), tandis que seul un chroniqueur soutient que « les frontières [entre les genres] sont relativement respectées» (P36). À ce propos, les répondants posent que les genres se mélangent lorsqu'on trouve «des commentaires personnels qui rentrent dans quelque chose qui devrait être objectif » (P35). Un autre chroniqueur juge, dans cette même optique, qu' « en nouvelle, en reportage, $[\ldots]$ tu es pas supposé mettre $[. .$.$] d'opinion » (P37).$

Les répondants s'appuient sur une norme de séparation (Burger, 2005, p. 224) qui repose sur la distinction entre genres factuels et d'opinion et selon laquelle il n'est pas permis d'insérer des marques de subjectivité dans les textes d'information. Les témoins réfèrent ici à un impératif jugé constitutif du journalisme nord-américain (Neveu, 2013 : 10), modèle normatif dans lequel tous les chroniqueurs déclarent s'inscrire et qu'ils opposent diamétralement au journalisme pratiqué en France : « tu lis un journal comme Libération, puis tu as toujours l'impression qu'il y a un commentaire éditorial quand tu lis une nouvelle, moi, j'aime pas ça » (P38). La dichotomie entre objectivité et subjectivité représente donc un repère normatif 
central pour les chroniqueurs, qui ne remettent pas en question la quête d'objectivité absolue dans les genres d'information, même si ce postulat du journalisme anglosaxon reste en réalité un but hors d'atteinte. Par exemple, dans la citation suivante, un chroniqueur condamne la transgression de la norme de l'objectivité, même s'il observe une certaine tolérance de son quotidien à propos de cette pratique :

Il y a pas d'opinion dans ces textes-là [la nouvelle], bon, à La Presse, on a quand même une certaine ouverture, mais quand même ... de voir ... qu'est-ce que j'ai lu récemment (?) ça m'a fait ... ah ben, mon Dieu, dans un texte de nouvelle avec une espèce de mot qui était, mais je sais plus c'est quoi ... alors, je peux pas vous le dire, mais ... mais effectivement, c'est quand même ... (P26)

Par ailleurs, un autre répondant estime que la séparation entre information et opinion peut lui demander un effort considérable en écriture de nouvelle, surtout quand il s'agit d'un sujet émotionnellement chargé. Aux yeux de ce chroniqueur, il est difficile de devoir renoncer au droit d'exprimer son opinion personnelle, privilège professionnel qui lui permettrait de se démarquer dans le champ journalistique :

Parfois le sujet est tellement incroyable, tu sais, tu es là comme journaliste, et puis tu vois à trois pas d'ici que ... une pauvre fille qu'est tombée sur le trottoir là, lors d'une manifestation, ça te révolte profondément, tu te dis c'est un être humain réel qu'est là, puis les trois policiers qui frappent sur elle, c'est quoi (?) c'est des fous ou quoi (?) alors là, si j'ai à faire une information, j'ai un problème là parce qu'il faut que je surmonte d'abord mon ... mon dégoût personnel, ma révolte, et puis ... mais je peux faire un billet à côté, c'est la façon de régler mon problème de surcharge émotive (P09)

L'alignement sur la norme de l'objectivité explique aussi que tous les chroniqueurs (sauf un) refusent de se considérer comme un modèle en matière de rédaction journalistique : « je veux pas vous dire, ils [les chroniqueurs] écrivent tous merveilleusement bien, y compris moi» (P27). Bien conscients de leur notoriété publique, les répondants mettent plutôt en évidence l'hétérogénéité de l'écriture journalistique pour insister sur l'importance de distinguer les genres factuels du commentaire. Les réponses obtenues à ce propos abondent dans le même sens :

Les chroniqueurs peuvent servir de modèles d'écriture en journalisme (?) pff ... un peu, mais [...] seulement dans une certaine mesure, c'est parce qu'ils sont dans un secteur tellement différent des autres, [...] qui a des limites et les limites viennent très vite, je suis pas sûr [...] que mes collègues vraiment, qui font de la nouvelle, peuvent vraiment s'inspirer de l'écriture des chroniqueurs (P25)

En outre, l'argument du respect de la norme de séparation permet à plusieurs témoins de conclure que les journaux mettent trop l'accent sur la promotion de la 
chronique, négligeant ainsi les autres genres : «ça [la chronique] bouffe l'espace pour la nouvelle et peut-être [...] les gens auraient besoin d'avoir plus de [...] faits bruts, plus de gens qui couvrent la nouvelle » (P36). De façon similaire, un autre témoin condamne la mise en scène des chroniqueurs encouragée par la direction des quotidiens et exige la revalorisation du travail du journaliste d'information, y compris celui des journalistes d'enquête et d'investigation. Ce répondant établit donc une opposition entre journalistes et chroniqueurs, tout en attribuant à ces deux groupes un statut égalitaire dans le champ journalistique :

Il y a beaucoup de jeunes journalistes qui veulent être chroniqueurs ... et comme on a fait $[. .$.$] des chroniqueurs, des ... des vedettes, des personnages publics, il y$ a des gens qui aspirent à ça, qui veulent être en avant, tout ça, mais certains n'ont pas le talent pour faire ça, [...] ce qui est mis en valeur, c'est la chronique, ben tout le monde tend vers ça, puis des fois $[\ldots]$ je trouve que c'est pas une bonne idée, $[\ldots]$ il faut revaloriser le travail de journaliste, $[\ldots]$ moi, je suis admiratif du travail de mes collègues qui font de l'enquête et tout ça, et puis il y en a qui adorent ça, puis ils ont pas de problème de ... se sentent pas lésés parce qu'ils sont pas le type de chroniqueur, mais je trouve qu'on a créé une espèce de culte de la chronique qui peut nuire à long terme à la profession (P38)

En outre, plusieurs chroniqueurs critiquent le nombre croissant de chroniqueurspigistes dans les tabloïds. S'ils distinguent ainsi les chroniqueurs à temps plein de ceux qui n'écrivent qu'occasionnellement à côté de leur profession régulière, il est intéressant de voir que ce sont les articles de ces derniers qui sont récusés, un peu comme si seuls les chroniqueurs dits professionnels avaient le droit de donner leur opinion :

Tout le monde fait de l'opinion, tu sais, Le Journal de Montréal, notre concurrent, ils embauchent pas des journalistes, mais ils embauchent des anciens politiques, tout le monde a une opinion sur tout, ça devient insupportable, même moi, je suis plus capable de me lire des fois là, [...] c'est pour ça que des fois je lâche la chronique et je fais du reportage parce que je suis plus capable d'avoir d'opinion là, il y en a trop (P19)

Enfin, un témoin affirme que le nombre de chroniques serait devenu tellement élevé que les chroniqueurs réussissent de moins en moins à se singulariser :

On est arrivé à un stade où il y a tellement de chroniques qu'elles perdent de l'impact parce qu'il y en a tellement que [...] les chroniqueurs ont de la difficulté à se distinguer les uns des autres, c'est ça le problème quand il y a beaucoup de chroniques, est-ce qu'il y en a trop (?) s'il y en avait trop, trop en vertu de quoi (?) si ça plait aux lecteurs, c'est sûr que les journaux vont continuer d'en ajouter, mais c'est sûr que c'est ... quand il y a énormément de chroniques, c'est plus difficile d'en avoir qui ont de l'impact (P25) 
Les chroniqueurs se montrent peu tolérants envers la transgression des genres journalistiques, surtout parce que la norme de l'objectivité est perçue comme un élément d'identification central avec le journalisme nord-américain qui, plus encore, contribue à assurer leur distance par rapport aux autres professionnels des médias. Par ailleurs, les témoins s'opposent à l'hybridation de leur propre genre, qui réside, bien entendu, non dans le mélange de traditions textuelles, mais dans l'ouverture de la chronique à des scripteurs venant de l'extérieur du champ journalistique. Les chroniqueurs expriment alors une certaine inquiétude concernant la diminution de l'impact de leurs textes sur les lecteurs et la spécificité de leur statut professionnel.

\section{Conclusion}

Les commentaires analysés nous amènent à conclure que les chroniqueurs considèrent pratiquer une profession distincte dans le paysage journalistique québécois. Ils tendent à percevoir les normes textuelles comme des constituants fondamentaux de leur propre identité professionnelle qui leur servent d'éléments de différenciation et de distinction par rapport à d'autres scripteurs provenant tant de l'intérieur que de l'extérieur du champ journalistique. Les chroniqueurs s'appuient sur des catégorisations génériques bien établies, reproduisant largement le système normatif en usage qui leur attribue le privilège de l'opinion personnelle, tout en plaidant en faveur d'une plus grande variation dans le journalisme d'information.

À l'inverse, lorsqu'il s'agit de se définir eux-mêmes en tant que groupe professionnel, les témoins se réfèrent moins aux normes textuelles sur lesquelles ils s'alignent pour mettre en avant le caractère individualisé de leurs chroniques, mais plutôt aux normes textuelles qui sont caractéristiques des autres genres journalistiques. Autrement dit, l'identité des chroniqueurs se construit davantage dans le rapport à l'autre, c'est-à-dire par une opposition à autrui et à ses pratiques professionnelles, que dans le regard posé sur leur propre groupe. Cela peut sans doute s'expliquer par le fait qu'une partie de la construction de l'identité des chroniqueurs provient essentiellement de la transgression délibérée qu'ils font de certaines normes textuelles bien établies, et ce pour mettre en scène leur propre personnalité sur le plan rédactionnel, d'où leur popularité médiatique. En ce sens, on peut interpréter la chronique comme le lieu où peuvent émerger de nouvelles pratiques d'écriture dans le champ journalistique, pratiques qui pourraient éventuellement être reprises par d'autres scripteurs. 


\section{Bibliographie}

Agnès, Y. (2008). Manuel de journalisme. Écrire pour le journal. Paris: La Découverte.

Amossy, R. (2010). La présentation de soi. Ethos et identité verbale (2è éd.). Paris : Presses Universitaires de France.

Aschenberg, H. (2003). Diskurstraditionen - Orientierungen und Fragestellungen. In H. Aschenberg \& R. Wilhelm (Eds.), Romanische Sprachgeschichte und Diskurstraditionen. Akten der gleichnamigen Sektion des XXVII. Deutschen Romanistentages (pp. 1-18). Tübingen : Gunter Narr.

Bourdieu, P. (1996). Sur la télévision. Paris : Liber.

Brin, C., Charron, J. \& de Bonville, J. (2004). Introduction. In C. Brin, J. Charron \& J. de Bonville (Eds.), Nature et transformation du journalisme. Théorie et recherches empiriques (pp. 1-31). Québec: Les Presses de 1'Université Laval.

Burger, H. (2005). Mediensprache. Eine Einführung in Sprache und Kommunikationsformen der Massenmedien (3è éd.). Berlin, New York: Walter de Gruyter.

Chalaby, J. (1998). The Invention of Journalism. London : McMillan Press.

Charaudeau, P. (2002). Contrat de communication. In P. Charaudeau \& D. Maingueneau (Eds.), Dictionnaire d'analyse du discours (pp. 138-141). Paris : Éditions du Seuil.

Dubar, C. (1991). La socialisation: construction des identités sociales et professionnelles. Paris : Armand Colin.

Gauger, H.-M. (1995). Über Sprache und Stil. München : Beck.

Gloy, K. (1993). Sprachnormforschung in der Sackgasse? Überlegungen zu Renate Bartsch, 'Sprachnormen : Theorie und Praxis'. Beiträge zur Geschichte der deutschen Sprache und Literatur, 115(1), 30-65.

Gloy, K. (2004). Norm. In U. Ammon (Ed.), Sociolinguistics. An International Handbook of the Science of Language and Society (pp. 392-399). Berlin, New York: Walter de Gruyter.

Grevisse, B. (2008). Écritures journalistiques. Stratégies rédactionnelles, multimédia et journalisme narratif. Bruxelles : De Boeck. 
74 Dynamiques de professionnalisation en communication

Koch, P. (1987). Distanz im Dictamen. Zur Schriftlichkeit und Pragmatik mittelalterlicher Brief-und Redemodelle in Italien. Thèse d'État, Universität Freiburg, Freiburg.

Maltais, R. (2010). L'écriture journalistique sous toutes ses formes. Montréal: Presses de l'Université de Montréal.

Meier, F. (2016). La perception des normes textuelles, communicationnelles et linguistiques en écriture journalistique. Une contribution à l'étude de la conscience linguistique des professionnels des médias écrits québécois. Thèse de doctorat, Universität Augsburg, Augsburg.

Neveu, E. (2013). Sociologie du journalisme (4è éd.). Paris : La Découverte.

Noël, A. (2009). Le style. Conseils pour écrire de façon claire et vivante. Montréal : Éditions La Presse.

Oesterreicher, W. (1997). Zur Fundierung von Diskurstraditionen. In B. Frank, T. Haye \& D. Tophinke (Eds.), Gattungen mittelalterlicher Schriftlichkeit (pp. 19-41). Tübingen ${ }^{\circ}$ Gunter Narr.

Picard, J.-C. (1999). La chronique dans les quotidiens québécois: un genre journalistique de plus en plus populaire. Les cahiers du journalisme, 6, 3649.

Pritchard, D. \& Sauvageau, F. (2008). Les journalistes canadiens. Un portrait de fin de siècle (6è éd.). Québec : Presses de l'Université Laval.

Ringoot, R. \& Utard, J.-M. (2005). Genres journalistiques et 'dispersion' du journalisme. In R. Ringoot \& J.-M. Utard (Eds.), Le journalisme en invention. Nouvelles pratiques, nouveaux acteurs (pp. 21-47). Rennes: Presses Universitaires de Rennes.

Ringoot, R. \& Utard, J.-M. (2009). Le genre: une catégorisation peu catégorique. In R. Ringoot \& J.-M. Utard (Eds.), Les genres journalistiques. Savoirs et savoir-faire (pp. 11-21). Paris : L'Harmattan.

Ross, L. (2005). L'écriture de presse. L'art d'informer (2è éd.). Montréal : Gaëtan Morin.

Ruellan, D. (1993). Le professionnalisme du flou. Identité et savoir-faire des journalistes français. Grenoble : Presses Universitaires de Grenoble.

Seiler, F. (2009). Adaptation sociolinguistique, hybridation et norme. In B. Pöll \& E. Schafroth (Eds.), Normes et hybridation linguistiques en francophonie. Actes de la section 6 du Congrès de l'Association des francoromanistes 
allemands, Augsbourg, 24-26 septembre 2008 (pp. 21-44). Paris : L'Harmattan.

Sormany, P. (2011). Le métier de journaliste (3è éd.). Montréal : Boréal.

Wilhelm, R. (2001). Diskurstraditionen. In M. Haspelmath, E. König, W. Oesterreicher \& W. Raible (Eds.), Language Typology and Language Universals. An International Handbook (pp. 467-477). Berlin, New York ${ }^{\circ}$ Walter de Gruyter. 